\title{
A PERCEPÇÃO DA QUALIDAdE dE VIDA DO IDOSO DISFÁGICO APÓS INTERVENÇÃO FONOAUDIOLÖGICA
}

\author{
Perceived quality of life of the dysphagic elderly \\ after swallowing therapy intervention
}

Paloma Alves Miquilussi ${ }^{1}$, Isabel de Lima Zanata ${ }^{2}$, Ana Paula Andrade Sartori ${ }^{3}$, Janaína Turcato Nonato da Silva ${ }^{4}$, Jasiel da Silva ${ }^{5}$

1. Fundação Estatal de Atenção em Saúde de Curitiba (FEAES). Residente da Residência Multiprofissional em Saúde do Idoso. ORCID: orcid.org/0000-0003-2847-8199

2. Fundação Estatal de Atenção em Saúde de Curitiba (FEAES). Coordenadora da Residência Multiprofissional em Saúde do Idoso. 2. ORCID: orcid.org/0000-0002-0902-5584

3. Fundação Estatal de Atenção em Saúde de Curitiba (FEAES). Tutora da Residência Multiprofissional em Saúde do Idoso. ORCID: orcid.org/0000-0002-9633-7905

4. Fundação Estatal de Atenção em Saúde de Curitiba (FEAES). Preceptora da Residência Multiprofissional em Saúde do Idoso. ORCID: orcid.org/0000-0002-7258-5146

5. Fundação Estatal de Atenção em Saúde de Curitiba (FEAES). Residente da Residência Multiprofissional em Saúde do Idoso. ORCID: orcid.org/0000-0003-1450-8529

CONTATO: Paloma Alves Miquilussi | Endereço: Rua Soldado Cipriano Pereira Bueno, 88 | Alto Boqueirão | Curitiba - Paraná | Telefone: (41) 99654-5112 | E-mail: fono_pa@yahoo.com.br

COMO CITAR: Miquilussi PA, Zanata IL, Sartori APA, Silva JTN, Silva J. A percepção da qualidade de vida do idoso disfágico após intervenção fonoaudiológica. R. Saúde Públ. 2019 Jul:2(1):93-102.

(c) COPYRIGHT Esta obra é disponibilizada nos termos da Licença Creative Commons - 4. 0

RESUMO Este trabalho teve o objetivo de identificar a percepção de qualidade de vida do idoso disfágico após intervenção fonoaudiológica. Trata-se de um estudo de caso longitudinal prospectivo de análise quantitativa, realizado com 4 pacientes encaminhados ao serviço ambulatorial de Fonoaudiologia de um hospital referência em saúde do idoso de Curitiba, os quais apresentavam queixa de disfagia e responderam ao protocolo SWAL-QOL antes e após programa de reabilitação. Foi calculada a média dos scores nos 11 domínios do protocolo nos dois momentos da pesquisa e em seguida foram analisados os resultados. Dos domínios avaliados no protocolo, 9 apresentaram 
aumento da média do score após terapia. Portanto, o estudo identificou mudança da percepção da qualidade de vida destes idosos, o que pode ser atribuído ao programa de reabilitação fonoaudiológica proposto aos participantes. No entanto, sugere-se mais pesquisas na área e com maior número de indivíduos.

PALAVRAS-CHAVE: Transtornos de Deglutição. Qualidade de Vida. Geriatria. Ciências da fala - Fonoaudiologia. Saúde do Idoso.

ABSTRACT This objective of this study was to identify in the percevedi quality of life of dysphagic elderly after swallowing therapy intervention. This is a prospective longitudinal case study of quantitative analysis, carried out whith 4 patients referred to a SpeechTherapy service in a reference hospital for elderly health in the city of Curitiba, who complained of dysphagia, and answered the SWAL-QOL protocol before and after a rehabilitation program. The mean scores were calculated in the 11 domains of the protocol in the two moments of the study, and the results were analyzed. Of the 11 domains evaluated by the protocol, 9 showed an increase in the mean score after therapy. Therefore, it was found that deglutition rehabilitation for dysphagic elderly patients may contribute to change the perception of the quality of life related to dysphagia. However, more research is needed in the area, and with a greater number of individuals.

KEYWORDS: Deglutition disorders. Quality of life. Geriatrics. Speech, Language and Hearing sciences - Speech therapy. Elderly health.

\section{INTRODUÇÃO}

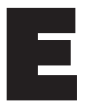
nvelhecer é um processo progressivo e natural que gera mudanças graduais mesmo nos indivíduos que gozem de boa saúde e hábitos saudáveis no decorrer de sua vida. Este processo é caracterizado pela capacidade do idoso se adaptar às mudanças fisiológicas decorrentes da idade, de forma a compensar essas limitações e alcançar satisfação pessoal mesmo diante de enfermidades características desta população. Cada indivíduo se adapta em menor ou maior grau, já que o bem-estar envolve critérios subjetivos, de acordo com suas expectativas individuais e seus potenciais psicológico e social'.

Estima-se que a partir de 2030, haverá uma inversão na pirâmide etária, com diminuição da população produtiva e predomínio da população idosa em relação à quantidade de crianças. Já em 2040, há projeções de que idosos cheguem a 23,8\% da população total ${ }^{2}$. O impacto na saúde e qualidade de vida destes indivíduos, considerando o aumento da prevalência de doenças crônicas, afeta de forma multidimensional esta população ${ }^{3}$.

Para a OMS, o termo "qualidade de vida" refere-se à percepção individual do ser humano quanto à sua posição na vida, objetivos e expectativas. É um termo de significado amplo e subjetivo, que engloba aspectos relativos à saúde física, psicológica, nível de independência, relações 
sociais, bem como quanto às suas atitudes frente às suas enfermidades ${ }^{4}$.

O distúrbio de deglutição associado ao processo natural do envelhecimento é definido como presbifagia. 0 idoso pode apresentar sinais e sintomas que comprometem esta função, tais como: acúmulo de alimento em cavidade oral, modificações na mastigação, escape de alimento durante a refeição, regurgitação nasal do alimento, tosse após a ingestão de alimento e/ou líquidos, odinofagia, engasgo, e sensação de bolo parado na região faríngea. Podem ocorrer como consequências destes sinais e sintomas: desnutrição, desidratação, aspiração laringotraqueal, pneumonias de repetição e aumento da morbimortalidade.

A avaliação da qualidade de vida relacionada à deglutição auxilia na mensuração do impacto que a disfagia pode trazer à vida do paciente ${ }^{5}$. Permite ainda verificar a percepção do paciente acerca de seu tratamento. Elaborado originalmente por Mchorney et al., o SWAL-QOL atualmente é o único protocolo validado para a língua portuguesa brasileira que avalia a qualidade de vida relacionada à deglutição, independentemente de sua etiologia6.

A intervenção fonoaudiológica no âmbito da disfagia consiste na realização de anamnese, avaliação indireta (sem oferta de alimento), a qual avalia a eficiência dos órgãos fonoarticulatórios envolvidos na deglutição; e avaliação direta ou funcional (com oferta de alimento), a qual tem o objetivo de observar o momento da alimentação do paciente, a fim de identificar sinais sugestivos de aspiração e estabelecer o grau de disfagia deste indivíduo. Por fim, auxilia no planejamento terapêutico, o qual engloba adequação da consistência da dieta, realização de manobras facilitadoras da deglutição e orientações ao paciente e familiares, objetivando criar condições para uma alimentação segura e capaz de garantir a nutrição e o prazer do indivíduo?.

Sendo assim, o objetivo deste trabalho é identificar a percepção de qualidade de vida do idoso disfágico após intervenção fonoaudiológica.

\section{METODOLOGIA}

Trata-se de um estudo de caso longitudinal prospectivo de análise quantitativa, realizado com pacientes encaminhados ao serviço ambulatorial de Fonoaudiologia de um hospital referência em saúde do idoso de Curitiba no período de março a agosto de 2018. Os idosos foram convidados a participar da pesquisa e os que aceitaram assinaram um Termo de Consentimento Livre e Esclarecido (TCLE). Como critérios de inclusão para a pesquisa foram considerados: pacientes com idade igual ou superior a 60 anos de ambos os sexos, que apresentavam queixa de disfagia, a qual foi confirmada através de avaliação clínica e condições cognitivas preservadas, que foram avaliadas através do discurso verbal. Como critérios de exclusão foram considerados: pacientes com idade inferior a 60 anos, ausência de queixa de disfagia no momento da abordagem, aqueles cujo diagnóstico não foi confirmado na avaliação clínica fonoaudiológica, pacientes sem condições cognitivas preservadas, bem como os que não aderiram ao programa de reabilitação proposto.

Este estudo foi aprovado pelo Comitê de Ética em Pesquisa da Secretaria de Saúde de Curitiba em 14 de dezembro de 2017, sob o parecer $n^{0} 2.439 .618$.

\section{AVALIAÇÃo CLÍNICA FONOAUDIOLÓGICA}

A primeira etapa consistiu na realização de avaliação clínica fonoaudiológica, a qual teve como instrumento o protocolo padronizado pela instituição. O objetivo desta avaliação foi verificar as condições de tonicidade, mobilidade e funcionalidade dos órgãos fonoarticulatórios, as condições clínicas do paciente, bem como foi possivel determinar o grau de disfagia dos pacientes da seguinte forma: Deglutição Normal, Deglutição Funcional, Disfagia Leve, Disfagia Moderada e Disfagia Grave ${ }^{8}$.

- Disfagia leve: a alimentação é mais 
demorada, podendo ocorrer episódios de engasgos. São orientadas refeições mais frequentes e em porções menores, maior atenção durante a deglutição, principalmente para líquidos, evitando escape precoce para a laringe.

- Disfagia moderada: dificuldade em iniciar a deglutição, podendo ocorrer penetração laríngea e/ou aspiração laringotraqueal. Presença de tosse, engasgos, pigarros e voz molhada. Utilizam-se manobras facilitadoras e posturais associadas à modificação da dieta e suplementação nutricional. O líquido deve ser engrossado. A via oral é preservada desde que a tosse seja eficaz.

- Disfagia grave: necessidade de alimentação suplementar, devido à dificuldade em manter uma ingestão hídrica e proteica calórica adequada, associada à dificuldade respiratória e ao pouco prazer alimentar. Indicação de via alternativa de alimentação exclusiva. Pode ocorrer aspiração laringotraqueal, inclusive de saliva.

\section{PROTOCOLO DE QUALIDADE DE VIDA (SWAL-QOL)}

Ao término da avaliação clínica fonoaudiológica, os pacientes responderam ao protocolo de Qualidade de Vida relacionado à disfagia SWALQOL. Trata-se de um protocolo de autoavaliação composto por 44 questões, divididas em onze domínios: deglutição como um fardo, desejo de se alimentar, duração da alimentação, frequência dos sintomas, seleção de alimentos, comunicação, medo de se alimentar, saúde mental, social, sono e fadiga. 0 paciente responde as questões levando em consideração a frequência em que os eventos acontecem (sempre, muitas vezes, algumas vezes, um pouco ou nunca). A pontuação de cada domínio varia de 0 a 100, sendo que quanto mais baixo o resultado obtido, pior é a qualidade de vida relacionada à deglutição ${ }^{6}$. Caso o paciente apresente algum impedimento físico para responder o protocolo, o pesquisador poderá auxiliar sem que o influencie em suas respostas.

Ao final desta etapa, iniciou-se um programa de reabilitação fonoaudiológica individualizada, levando em consideração as queixas e o resultado da avaliação clínica de cada paciente. Este programa terapêutico teve duração de até 10 sessões, sendo que cada sessão durava 40 minutos.

Durante as sessões foram utilizadas como estratégias terapêuticas: exercícios de fortalecimento das estruturas responsáveis pelas funções de mastigação e deglutição, estimulação sensorial, adaptação da consistência alimentar, utilização de posturas e manobras compensatórias que auxiliam a deglutição, além de orientações ao paciente e familiares. Os recursos terapêuticos utilizados foram escolhidos conforme a necessidade de cada paciente e dados da avaliação clínica destes.

Ao término do programa de reabilitação, o protocolo SWAL-QOL foi reaplicado, a fim de comparar a percepção de qualidade de vida do idoso disfágico antes e após intervenção fonoaudiológica.

\section{RESULTADOS}

Dos pacientes encaminhados ao ambulatório de fonoaudiologia no período do estudo, sete se enquadraram nos critérios da pesquisa, no entanto, somente quatro finalizaram todas as suas etapas. Dois dos pacientes eram do sexo feminino e dois do sexo masculino. A média de idade foi de 72 anos, sendo que a idade mínima foi de 61 anos e a máxima 85 anos.

Todos os pacientes se alimentavam exclusivamente por via oral, e em relação à classificação da disfagia, antes do programa de reabilitação três pacientes apresentavam grau de disfagia leve e uma moderada. Após a intervenção, dois apresentaram deglutição funcional, um disfagia leve e um disfagia moderada. 
A população de idosos participantes da pesquisa, bem como seus dados relacionados à alimentação nos períodos pré e pós-intervenção fonoaudiológica estão caracterizados nos quadros abaixo:

Quadro 1 Caracterização dos idosos participantes da pesquisa pré-intervenção fonoaudiológica.

\begin{tabular}{|c|c|c|c|c|c|c|}
\hline PACIENTE & SEXO & IDADE & $\begin{array}{l}\text { DOENÇA DE } \\
\text { BASE }\end{array}$ & $\begin{array}{l}\text { QUEIXA } \\
\text { PRINCIPAL }\end{array}$ & $\begin{array}{c}\text { VIA DE } \\
\text { ALIMENTAÇÃO } \\
\text { PRÉ-INTERVENÇÃO }\end{array}$ & $\begin{array}{l}\text { CONSISTÊNCIA } \\
\text { DA DIETA PRÉ- } \\
\text { INTERVENÇÃO }\end{array}$ \\
\hline 1 & M & 85 & AVC & Engasgo & Via oral exclusiva & Branda e líquida \\
\hline 2 & M & 65 & AVC & $\begin{array}{c}\text { Tosse, bólus faríngeo, } \\
\text { odinofagia }\end{array}$ & Via oral exclusiva & Branda e líquida \\
\hline 3 & $\mathrm{~F}$ & 81 & AVC & Engasgo & Via oral exclusiva & Branda e líquida \\
\hline 4 & $\mathrm{~F}$ & 62 & $\begin{array}{l}\text { Fibromialgia, } \\
\text { Paralisia de Bell }\end{array}$ & $\begin{array}{l}\text { Bólus faríngeo, } \\
\text { engasgo }\end{array}$ & Via oral exclusiva & Branda e líquida \\
\hline
\end{tabular}

${ }^{* *}$ Legenda: AVC: Acidente Vascular Cerebral

Fonte: Elaborado pelos autores (2018)

Quadro 2 Caracterização dos achados de avaliação, grau de disfagia antes e após intervenção e estratégias terapêuticas utilizadas no processo de reabilitação fonoaudiológica.

\begin{tabular}{|c|c|c|c|c|}
\hline PACIENTE & $\begin{array}{l}\text { ACHADOS DA } \\
\text { AVALIAÇÃO }\end{array}$ & $\begin{array}{l}\text { GRAU DA DISFAGIA } \\
\text { PRÉ-INTERVENÇÃO }\end{array}$ & $\begin{array}{c}\text { ESTRATÉGIAS TERAPÊUTICAS } \\
\text { UTILIZADAS }\end{array}$ & $\begin{array}{l}\text { GRAU DA DISFAGIA } \\
\text { PÓS- INTERVENÇÃOO }\end{array}$ \\
\hline 1 & $\begin{array}{l}\text { Sinais sugestivos de } \\
\text { penetração laríngea } \\
\text { para consistência } \\
\text { líquida }\end{array}$ & $\begin{array}{l}\text { Disfagia } \\
\text { Moderada }\end{array}$ & $\begin{array}{c}\text { Exercícios miofuncionais } \\
\text { ativos, estimulação sensorial } \\
\text { extra e intra-orais, adequação } \\
\text { da consistência alimentar. } \\
\text { manobras facilitadoras de } \\
\text { deglutição, orientações gerais } \\
\text { ao paciente e familiares. }\end{array}$ & $\begin{array}{l}\text { Disfagia } \\
\text { Moderada }\end{array}$ \\
\hline 2 & $\begin{array}{l}\text { Ausência de sinais } \\
\text { sugestivos de } \\
\text { penetração laríngea/ } \\
\text { broncoaspiração }\end{array}$ & Disfagia Leve & $\begin{array}{l}\text { Exercícios miofuncionais } \\
\text { ativos, estimulação sensorial } \\
\text { extra e intra-orais, orientações } \\
\text { gerais ao paciente e familiar. }\end{array}$ & Disfagia Leve \\
\hline 3 & $\begin{array}{l}\text { Ausência de sinais } \\
\text { sugestivos de } \\
\text { penetração laríngea/ } \\
\text { broncoaspiração }\end{array}$ & Disfagia Leve & $\begin{array}{c}\text { Exercícios miofuncionais } \\
\text { ativos, estimulação sensorial } \\
\text { extra e intra-orais, orientações } \\
\text { gerais à paciente e familiar. }\end{array}$ & $\begin{array}{l}\text { Deglutição } \\
\text { Funcional }\end{array}$ \\
\hline 4 & $\begin{array}{l}\text { Ausência de sinais } \\
\text { sugestivos de } \\
\text { penetração laríngea/ } \\
\text { broncoaspiração }\end{array}$ & Disfagia Leve & $\begin{array}{c}\text { Exercícios miofuncionais } \\
\text { ativos, estimulação sensorial } \\
\text { extra e intra-orais, orientações } \\
\text { gerais à paciente. }\end{array}$ & $\begin{array}{l}\text { Deglutição } \\
\text { Funcional }\end{array}$ \\
\hline
\end{tabular}

Fonte: Elaborado pelos autores (2018) 
O desempenho individual dos pacientes por domínio avaliado através do protocolo SWAL-QOL está apresentado na tabela a seguir:

Tabela 1 Scores obtidos em cada domínio do SWAL-QOL, antes e após intervenção fonoaudiológica

\begin{tabular}{|c|c|c|c|c|c|c|c|c|}
\hline & \multicolumn{2}{|c|}{ Paciente 1} & \multicolumn{2}{|c|}{ Paciente 2} & \multicolumn{2}{|c|}{ Paciente 3} & \multicolumn{2}{|c|}{ Paciente 4} \\
\hline & Pré & Pós & Pré & Pós & Pré & Pós & Pré & Pós \\
\hline Deglutição como um fardo & 75 & 100 & 87,5 & 37,5 & 50 & 75 & 25 & 0 \\
\hline Desejo de se alimentar & 66,66 & 100 & 58,33 & 66,66 & 75 & 83,33 & 16,66 & 33,33 \\
\hline Duração da alimentação & 75 & 100 & 25 & 25 & 0 & 25 & 0 & 25 \\
\hline Frequência de sintomas & 73,21 & 89,28 & 42,85 & 58,92 & 30,35 & 64,28 & 26,78 & 46,42 \\
\hline Seleção de alimentos & 100 & 100 & 75 & 50 & 12,5 & 12,5 & 12,5 & 25 \\
\hline Comunicação & 100 & 100 & 62,5 & 87,5 & 37,5 & 75 & 12,5 & 12,5 \\
\hline Medo de se alimentar & 87,5 & 100 & 43,75 & 37,5 & 0 & 56,25 & 25 & 50 \\
\hline Saúde mental & 100 & 100 & 40 & 85 & 0 & 80 & 0 & 50 \\
\hline Social & 100 & 100 & 15 & 35 & 5 & 25 & 0 & 25 \\
\hline Sono & 50 & 25 & 75 & 100 & 0 & 50 & 0 & 0 \\
\hline Fadiga & 100 & 100 & 8,33 & 33,33 & 16,66 & 25 & 8,33 & 50 \\
\hline
\end{tabular}

Fonte: Elaborado pelos autores (2018)

Dos 11 domínios que o SWAL-QOL avalia, 9 apresentaram média superior após reabilitação fonoaudiológica em relação à média obtida antes da intervenção, como demonstra a tabela abaixo:

Tabela 2 Representação da média dos scores obtidos através do protocolo SWAL-QOL pré e pós-intervenção fonoaudiológica

\begin{tabular}{|c|c|c|}
\hline & Média Pré-intervenção & Média Pós-intervenção \\
\hline Deglutição como um fardo & 59,37 & 53,12 \\
\hline Desejo de se alimentar & 54,16 & 70,83 \\
\hline Duração da alimentação & 25 & 43,75 \\
\hline Frequência de sintomas & 43,29 & 64,72 \\
\hline Seleção de alimentos & 50 & 46,87 \\
\hline Comunicação & 53,12 & 68,75 \\
\hline Medo de se alimentar & 39,06 & 60,93 \\
\hline Saúde mental & 35 & 78,75 \\
\hline Social & 30 & 46,25 \\
\hline Sono & 31,25 & 43,75 \\
\hline Fadiga & 33,33 & 52.08 \\
\hline
\end{tabular}

Fonte: Elaborado pelos autores (2018) 


\section{DISCUSSÃo}

Os resultados obtidos na pesquisa têm limitações, já que não foi possível realizar análise estatística pelo número pequeno de casos estudados. Para comparar os resultados de forma significativa seriam necessários testes. Portanto, o que se observou foi uma melhora clínica de alguns scores pré e pós-intervenção.

Neste estudo, a média dos scores obtidos antes da intervenção fonoaudiológica no domínio "Deglutição como um Fardo" do protocolo SWALQOL apresentou mudança após intervenção. Este resultado pode estar relacionado ao fato de que a alimentação do idoso é influenciada por fatores biológicos, psicológicos, sociais e culturais. A aposentadoria, viuvez ou a saída dos filhos de casa estão relacionadas à mudança de suas práticas alimentares. A falta de pessoas à sua volta para partilhar refeições faz com que o individuo se sinta menos motivado a se alimentar e preparar suas próprias refeições ${ }^{9}$. Além disso, o edentulismo está relacionado diretamente com a manutenção da qualidade de vida dos idosos, pela limitação que a perda dos dentes acarreta à função de mastigar e o impacto na sua nutrição, autoestima e integração social ${ }^{10}$.

O domínio "Desejo de se alimentar" apresentou diferença nos dois momentos da aplicação do questionário. Tal achado pode ser justificado pelo fato de que os sintomas da disfagia influenciam de forma negativa o apetite e o prazer do idoso em se alimentar11. Logo, a reabilitação fonoaudiológica, através de estratégias terapêuticas, resulta em alterações fisiológicas no mecanismo de deglutição, proporcionando melhora desta função que sofre mudanças funcionais no processo de envelhecer ${ }^{12}$. Sendo assim, a melhora da disfagia após a terapia realizada com os idosos do estudo pode ter influenciado na melhora do desejo em se alimentar.

No decorrer do processo de envelhecimento, as funções estomatognáticas ficam mais lentas e descoordenadas, isso se deve à diminuição da força mastigatória, perda dos dentes ou pela má adaptação das próteses dentárias. Estes fatores levam o idoso necessitar de mais tempo para completar sua refeição ${ }^{13}$. Estas modificações podem atingir de forma negativa a qualidade de vida deste indivíduo ${ }^{14}$. Neste estudo, os idosos pesquisados apresentaram mudança do score no domínio "Duração da Alimentação" antes e após intervenção, este fato pode ser justificado pelo possivel ganho de força e mobilidade dos órgãos fonoarticulatórios após intervenção fonoaudiológica, auxiliando assim o idoso a se alimentar de forma mais ágil.

O domínio "Frequência dos sintomas" também apresentou modificação dos scores nos idosos pesquisados, ou seja, houve diminuição da frequência dos sintomas de disfagia após realização das sessões de terapia fonoaudiológica. Isso pode ser justificado pelo fato de a intervenção fonoaudiológica contribuir para a melhora da qualidade de vida do paciente com distúrbio de deglutição ${ }^{15}$. A realização de exercícios que envolvam a musculatura responsável pela deglutição, além de proporcionar o fortalecimento destas estruturas, recruta unidades motoras a nível central16.

No processo de envelhecimento, seja este senil ou senescente, há prejuízo nas estruturas do sistema sensório-motor oral e consequentemente das funções por eles exercidas ${ }^{17}$. Com isso, o idoso ao longo deste processo realiza adaptações e estratégias para facilitar sua alimentação, como, por exemplo, substituir alimentos mais duros e fibrosos pelos mais macios e umidificados ${ }^{13}$. Além disso, o prejuízo dos aspectos sensoriomotores e a redução da sensibilidade gustativa presente nesta população podem interferir na seleção dos alimentos destes indivíduos ${ }^{18}$. A alteração do score após intervenção no domínio "Seleção dos Alimentos" encontrado neste estudo pode ser relacionado ao fato dos déficits citados acima serem características predominantes nessa população, ou então ser necessário um programa de intervenção mais prolongado.

Durante o processo de envelhecimento, o 
idoso apresenta modificação das habilidades de comunicação relacionada à voz, a qual definimos como presbifonia. Essa característica é inerente à fisiologia do envelhecimento, no entanto ela pode afetar a qualidade de vida do idoso ${ }^{19}$. Queixas como rouquidão, soprosidade vocal e diminuição do volume da voz são comuns nesta população e isso ocorre devido ao enfraquecimento das estruturas do trato vocal ${ }^{20}$. Apesar da habilidade de comunicação não estar diretamente ligada à de deglutir, o domínio "Comunicação" apresentou modificação após intervenção fonoaudiológica. Isso pode ser justificável pelo fato da produção vocal e a função de deglutição envolverem estruturas em comum, justificando o aprimoramento de ambas as funções após realização de exercícios que condicionem os músculos envolvidos ${ }^{21}$.

O domínio "Medo de se alimentar" apresentou mudança neste estudo, porém pouco foi encontrado na literatura sobre esta questão. No entanto, em um estudo realizado através de entrevistas com idosos institucionalizados, os quais eram independentes e tinham cognitivo preservado, identificou-se 20 idosos com queixas relacionadas à deglutição. Essas entrevistas foram categorizadas, sendo que a categoria mais marcante do estudo, por ter englobado todos os indivíduos entrevistados, foi a de sentimentos e sensações que a dificuldade de deglutição trazia a estes idosos. Foi identificado em todos os entrevistados o sofrimento de conviver com o problema de deglutição, devido ao medo de engasgar e à falta de ar presente durante as refeições, pois cada um deles via o sintoma de engasgar de forma negativa e ligado à sua finitude como indivíduo 22 .

As alterações na dinâmica da deglutição podem acarretar respostas psicossociais como ansiedade, medo, insegurança e redução da sua autoestima, devido às modificações sociais relacionadas à alimentação, impactando diretamente em sua qualidade de vida e influenciando de forma negativa o apetite do idoso ${ }^{23}$. Além disso, a comunicação na terceira idade sofre grande impacto. $\mathrm{O}$ idoso pode apresentar dificuldade de falar e se expressar, fato que dificulta sua integração social, levando este indivíduo ao isolamento social por sentimentos de solidão e tristeza ${ }^{24}$. O domínio "Saúde Mental" foi o que apresentou maior mudança neste estudo, isso pode estar relacionado ao fato de que a alimentação e a comunicação da população deste estudo no geral foram aprimoradas, levando assim a mudanças emocionais nestes indivíduos.

Resultados semelhantes foram obtidos no domínio "Social". Não é incomum que o idoso que apresenta desordem na função da deglutição perca o prazer em se alimentar e se isole socialmente devido a este fator, afetando de forma significativa seu bem-estar e percepção de qualidade de $v^{\text {vida }}{ }^{25}$. Além disso, mais uma vez a comunicação se faz presente, se considerarmos que idosos com dificuldades nesta habilidade tendem a se isolar socialmente, fato que interfere em sua qualidade de vida ${ }^{26}$. Como citado acima, isso pode também ser relacionado à mudança geral dos scores pósintervenção, bem como relacionado à alteração no domínio "Comunicação".

O processo de envelhecimento por si só acarreta prejuizo na eficiência do sono do idoso, porém o uso de certas medicações e a necessidade de ir ao banheiro com frequência no período noturno, por exemplo, são alguns dos fatores responsáveis por agravar a má qualidade do sono e interferir no desempenho das atividades de vida diária deste indivíduo ${ }^{27}$. Apesar do sono não estar diretamente ligado à qualidade da alimentação, este distúrbio é uma das queixas mais frequentes referidas pelo idoso, já que gera um grande impacto na sua percepção de qualidade de vida ${ }^{28}$. 0 domínio "Sono" foi o que mais também apresentou alteração neste estudo.

Pensando no idoso sarcopênico, a diminuição de força e a fadiga são sintomas associados à baixa imunidade e redução da qualidade de vida ${ }^{29}$. Houve mudança no domínio "Fadiga" após intervenção. Isso pode ser relacionado ao fato da melhora do domínio "Saúde Mental", já que uma das queixas do idoso fragilizado emocionalmente é a sensação de extrema fadiga. ${ }^{30}$ 
A avaliação de qualidade de vida é o indicador mais utilizado para investigar o bem-estar físico e mental da saúde do idoso. Muitas vezes o envelhecimento é considerado, pelo próprio idoso, como um processo de constante declínio, fato que afeta a percepção de seu estado de saúde ${ }^{31}$. A forma subjetiva de o idoso julgar sua condição de saúde sofre interferência de sua experiência pessoal, das informações repassadas por profissionais da saúde, bem como pela observação de seu próprio comportamento e de idosos de sua convivência ${ }^{32}$.

\section{CONCLUSÃO}

O presente trabalho identificou mudanças na percepção da qualidade de vida de idosos disfágicos atendidos no ambulatório de um hospital referência em saúde do idoso de Curitiba. Este resultado pode ser atribuído ao programa de reabilitação fonoaudiológica, o qual foi proposto aos participantes. No entanto, sugerem-se mais pesquisas na área e com maior número de indivíduos.

\section{REFERÊNCIAS}

1. Faller JW, Teston EF, Marcon SS. Estrutura conceptual do envelhecimento em diferentes etnias. Rev Gaúch Enferm. [Internet]. 2018 [citado 2018 Nov 12;39:e66144. Disponivel em : http://www. scielo.br/pdf/rgenf/v39/1983-1447-rgenf-39-e66144.pdf.

2. Zen D, Leite MT, Hildebrandt LM, Silva LAA, Van der Sand ICP. Políticas de atenção a idosos na voz de gestores municipais de saúde. Rev Gaúch Enferm. [Internet 2018 [citado 2018 Ago 30]:39:e62502. Disponivel em: http://www.scielo.br/pdf/rgenf/ v39/1983-1447-rgenf-39-e62502.pdf.

3. Silveira MB, Saldanha RP, Leite JC, Silva TO, Silva T, Filippin LI. Construção e validade de conteúdo de um instrumento para avaliação de quedas em idosos. Einstein (São Paulo). [Internet]. 2018 [citado 2018 Dez 1];16(2):eA04154. Disponivel em: http:// www.scielo.br/pdf/eins/v16n2/pt_1679-4508-eins-16-02-eA04154.pdf.

4. Domingues JP, Oliveira DC, Marques SC. Representações sociais da qualidade de vida de pessoas que vivem com HIV/AIDS. Texto contexto - enferm.. [Internet]. 2018 [citado 2018 Dez 2]:27(2):e1460017. Disponivel em: http://www.scielo.br/pdf/tce/ v27n2/0104-0707-tce-27-02-e1460017.pdf.
5. Silva BF, Finard SA, Olchik MR. Qualidade de vida em pacientes com doença de Machado-Joseph sob acompanhamento fonoaudiológico para disfagia. Rev CEFAC [Internet]. 2016 Jul-Ago [citado 2018 Out 31]; 18(4):992-1000. Disponivel em: http://www. scielo.br/pdf/rcefac/v18n4/1982-0216-rcefac-18-04-00992.pdf.

6. Portas JG. Validação para a língua portuguesa brasileira dos questionários: qualidade de vida em disfagia (SWAL-QOL) e satisfação do paciente e qualidade do cuidado no tratamento da disfagia (SWAL-CARE) [tese]. São Paulo: Fundação Antônio Prudente; 2009 .

7. Turra GS. Intervenção fonoaudiológica em pacientes com disfagia, pós intubados e sem morbidades neurológicas [tese na internet]. Porto Alegre: Universidade Federal do Rio Grande do Sul; 2013 [citado 2018 Out 31]. Disponivel em: https://lume.ufrgs.br/ handle/10183/97953 y.

8. Najas M, coordenadora. I Consenso Brasileiro de Nutrição e Disfagia em Idosos Hospitalizados. Sociedade Brasileira de Geriatria e Gerontologia [Internet]. Barueri, SP. Minha Editora; 2011. [citado 2018 Nov 5]. Disponível em: https://sbgg.org.br//wp-content/uploads/2014/10/Consenso_Brasileiro_de_Nutricao1.pdf.

9. Cavalcante CMS, Carvalho MCVS, Ferreira FR, Prado SD. Sentidos da alimentação fora do lar para homens idosos que moram sozinhos. Rev Bras Geriatr Gerontol [Internet]. 2015 [citado 2018 Nov 10]:18(3):611-620. Disponivel em: http://www.scielo.br/pdf/ rbgg/v18n3/1809-9823-rbgg-18-03-00611.pdf.

10. Agostinho ACMG, Campos ML, Silveira JLGC. Edentulismo, uso de prótese e autopercepção de saúde bucal entre idosos. Rev Odontol UNESP. [Internet]. 2015 Mar.-Apr [citado 2018 Nov 30]:44(2):74-79. Disponivel em: http://www.scielo.br/pdf/rounesp/ v44n2/1807-2577-rounesp-44-2-74.pdf.

11. Nogueira LR, Morimoto JM, Tanakac JAW, Bazanelli AP. Avaliação qualitativa da alimentação de idosos e suas percepções de hábitos alimentares saudáveis. J Health Sci. [Internet] 2016 [citado 2018 Dez 2]:18(3):163-0. Disponivel em: http://www.pgsskroton. com.br/seer/index.php/JHealthSci/article/view/3119/3299

12. Crary MA, Carnaby GD. Adoption into clinical practice of two therapies to manage swallowing disorders: exercise based swallowing reabilitation and electrical stimulation. Curr Opin Otoryngol Head Neck Surg. [Internet]. 2014 June [citado 2018 Dez 3]:22(3):172-80. Disponível em: https://www.ncbi.nlm.nih.gov/pmc/ articles/PMC4104745/pdf/nihms604730.pdf.

13. Oliveira BS, Delgado SE, Brescovici SM. Alterações das funções de mastigação e deglutição no processo de alimentação de idosos institucionalizados. Rev Bras Geriatr Gerontol. [Internet] 2014 [citado 2018 Dez 3]:17(3):575-58. Disponivel em: http://www.scielo. br/pdf/rbgg/v17n3/1809-9823-rbgg-17-03-00575.pdf

14. Souza IAL, Massi G. A saúde fonoaudiológica a partir do discurso do idoso institucionalizado. Rev CEFAC. [Internet]. 2015 Jan-Fev [citado 2018 Dez 6]; 17(1):300-307. Disponível em: http:// www.scielo.br/pdf/rcefac/v17n1/1982-0216-rcefac-17-01-00300. pdf.

15. Sousa IMCB, Vasconcelos VECBC, Ferraz PRR. Reabilitação fonoaudiológica da disfagia orofaríngea após acidente vascular encefálico: revisão de literatura. Journal of Specialist [Internet] 2018 [citado 2018 Dez 6]:1(1):1-11. Disponivel em: http://journalofspecialist.com/jos/index.php/jos/article/view/65/29. 
16. Alves ICF. Programa terapêutico fonoaudiológico ambulatorial para disfagia orofaríngea em adultos e idosos [tese na internet]. São Paulo: Faculdade de Medicina da Universidade de São Paulo; 2015 [citado 2018 Dez 6]. Disponível em: http://www.teses.usp.br/ teses/disponiveis/5/5170/tde-12012016-110116/pt-br.php.

17. Olchik MR, Ayres A, Signorini AV, Flores LS. Impacto das alterações das estruturas do sistema estomatognático na deglutição de idosos acamados. RBCEH [Internet]. 2016 [citado 2018 Dez 4]:13(2):135-42. Disponivel em: http://seer.upf.br/index.php/rbceh/ article/view/5673/pdf.

18. Junior HVM. Evidências de validade do questionário autorreferido para rastreamento de disfagia orofaríngea em idosos - RADI [tese na internet ]. Natal: Universidade Federal do Rio Grande do Norte; 2018 [citado 2018 Dez 4]. Disponível em: https://repositorio.ufrn.br/jspui/bitstream/123456789/25636/1/ HipolitoVirgilioMagalhaes_unior_TESE.pdf

19. Chiossi JSC, Roque FP, Goulart BNG, Chiari BM. Impacto das mudanças vocais e auditivas na qualidade de vida de idosos ativos. Ciênc Saúde Colet [Internet]. 2014 [citado 2018 Nov 28]:19(8):3335-42. Disponivel em: http://www.scielo.br/pdf/csc/ v19n8/1413-8123-csc-19-08-03335.pdf

20. Vilanova JR, Almeida CPB, Goulart, BNG. Distúrbios fonoaudiológicos autodeclarados e fatores associados em idosos. Rev. CEFAC. [Internet]. 2015 Mai-Jun [citado 2018 Dez 1]:17(3):72026. Disponivel em: http://www.scielo.br/pdf/rcefac/v17n3/19820216-rcefac-17-03-00720.pdf.

21. Alves ICF, Andrade CRF. Mudança funcional no padrão de deglutição por meio da realização de exercícios orofaciais. CoDAS [Internet]. 2017 [citado 2018 Dez 5];29(3):e20160088. Disponível em: http://www.scielo.br/pdf/codas/v29n3/2317-1782-codas-293-e20160088.pdf.

22. Rozenfeld M. A percepção do engasgo por idosos institucionalizados. Estud Interdiscip Envelhec [Internet]. 2005 [citado 2018 Nov 16];7:69-85. Disponivel em: https://seer.ufrgs.br/RevEnvelhecer/article/viewFile/4758/2673

23. Gaspar MRF, Pinto GS, Gomes RHS, Santos RS, Leonor VD. Avaliação da qualidade de vida em pacientes com disfagia neurogênica. Rev CEFAC [Internet]. 2015 Nov-Dez [citado 2018 Dez 1]:17(6):1939-45. Disponivel em: http://www.scielo.br/pdf/rcefac/ v17n6/1982-0216-rcefac-17-06-01939.pdf

24. Santiago, LM, Graça CML, Rodrigues MCO, Santos GB. Caracterização da saúde de idosos numa perspectiva fonoaudiológica. Rev CEFAC [Internet]. 2016 Set-Out [citado 2018 Nov 26];18(5):1088-96. Disponivel em: http://www.scielo.br/pdf/rcefac/v18n5/1982-0216-rcefac-18-05-01088.pdf.

25. Nishida AEN. Associação entre distúrbios de deglutição e estado nutricional em idosos da comunidade [tese na internet]. Campinas: Universidade Estadual de Campinas; 2018 [citado 2019 Maio 13. Disponivel em: http://repositorio.unicamp.br/jspui/bitstream/ REPOSIP/332383/1/Nishida_AlineEliasDoNascimento_M.pdf.

26. Chiossi JSC, Roque FP, Goulart BNG, Chiari BM. Impacto das mudanças vocais e auditivas na qualidade de vida de idosos ativos. Ciênc Saúde Colet [Internet]. 2014 [citado 2018 Dez 3];19(8):333542. Disponivel em: http://www.scielo.br/pdf/csc/v19n8/1413-8123csc-19-08-03335.pdf.

27. Napoleão M. Monteiro B, Espirito-Santo H. Qualidade sub- jetiva do sono, sintomas depressivos, sentimentos de solidão e institucionalização em pessoas idosas. Rev Port Invest Comport Soc [Internet]. 2016 [citado 2018 Dez 5]:2(2):12-24. Disponível em: https://rpics.ismt.pt/index.php/ISMT/article/view/37/pdf.

28. Mansano-Schlosser TC, Santos AA, Camargo-Rossignolo SO, Freitas DCCV, Lorenz VR, Ceolim MF. Idosos institucionalizados: organização cronológica das rotinas diárias e qualidade do sono. Rev Bras Enferm [Internet]. 2014 Jul-Ago [citado 2018 Dez 4]:67(4):610-6. Disponivel em: http://www.scielo.br/pdf/reben/ v67n4/0034-7167-reben-67-04-0610.pdf.

29. Sousa KT, Mesquita LAS, Pereira LA, Azeredo CM. Baixo peso e dependência funcional em idosos institucionalizados de Uberlândia (MG), Brasil. Ciênc Saúde Colet [Internet]. 2014 [citado 2018 Nov 29]:19(8):3513-20. Disponivel em: http://www.scielo.br/pdf/ csc/v19n8/1413-8123-csc-19-08-03513.pdf.

30. Aguiar LS, Santos WL. Conhecimento dos enfermeiros quanto ao tratamento da depressão na terceira idade. REVISA [Internet]. 2014 Jul-Dez [citado 2018 Dez 3];3(2):133-40. Disponível em: http://revistafacesa.senaaires.com.br/index.php/revisa/article/ view/133/78

31. Sousa ACSN, Sousa RASA. Contribuições da fonoaudiologia para o envelhecimento ativo estudo de caso: idosa de 78 anos. Rev Por Divul [Internet]. 2012 [citado 2018 Dez 16]:2(23):37-46. Disponivel em: http://www.portaldoenvelhecimento.com/revistanova/index.php/revistaportal/article/viewFile/541/579.

32. Melo DM, Falsarella GR, Neri AL. Autoavaliação de saúde, envolvimento social e fragilidade em idosos ambulatoriais. Rev Bras Geriatr Gerontol [Internet]. 2014 [citado 2018 Nov 10];17(3):47184. Disponivel em http://www.scielo.br/pdf/rbgg/v17n3/18099823-rbgg-17-03-00471.pdf. 